\title{
Optimal Pricing in Stochastic Scalable Video Coding Multicasting System
}

\author{
Chih-Yu Wang ${ }^{1,2}$, Yan Chen ${ }^{2}$, Hung-Yu Wei ${ }^{1}$, K. J. Ray Liu ${ }^{2}$ \\ ${ }^{1}$ Graduate Institute of Communication Engineering, National Taiwan University, Taipei, Taiwan \\ ${ }^{2}$ Electrical and Computer Engineering, University of Maryland, College Park
}

\begin{abstract}
Heterogeneous multimedia content delivery over wireless networks is an important yet challenging issue. A promising solution is combining multicasting and scalable video coding (SVC) techniques via cross-layer design which has been shown to be effectively solution in the literature. Nevertheless, most existing works on SVC multicasting system focus on the static scenarios. In addition, the economic value of SVC multicasting system has seldom been explored. In this work, we study a subscriptionbased SVC multicasting system with stochastic user arrival and heterogeneous user preferences. A stochastic framework based on Multi-dimensional Markov Decision Process (M-MDP) is proposed to study the negative network externality existing in the proposed system. A game-theoretic analysis is conducted to understand the rational demands from heterogeneous users the subscription economic model. We show that the optimal pricing strategy which maximizes the expected revenue of the service provider can be derived through dynamic iterative updating techniques. Moreover, the overall user's valuation on the system is maximized under such an optimal pricing strategy. Finally, the solution efficiency is evaluated through simulations.
\end{abstract}

\section{INTRODUCTION}

With the development of multimedia compression and the advance of wireless networking techniques, multimedia content delivery over wireless networks becomes more and more popular. One challenging issue in such systems is how to maintain the quality of service due to the scare resource in wireless networks. Multicasting in wireless communications is a natural solution to this challenge. The service provider categorizes users into multicasting groups according to their demands in contents, and then perform multicasting in delivering. However, heterogeneous users may use devices with different computational capabilities. The service provider may need to deliver the same content in multiple qualities. This introduces serious redundancy in the delivery and therefore further aggravates the overloading issue.

Scalable video coding (SVC) is a promising technique to resolve the content redundancy issue [1]. It encodes the videos into a series of layers. A receiver may derive a low quality video by decoding the base layer alone, or a higher quality video by decoding the base layer and subsequent enhancement layers. By combining multicasting and SVC techniques, the cross-layer design shows great potentials in enhancing the quality of multimedia delivery service.

The utility-based approach is a popular approach to the cross-layer SVC multicasting system [2], where users derive some utilities if they receive and decode the demanding videos correctly. Generally, the wireless multicast resource allocation problem is NP-hard [3]. Therefore, approximate greedy algorithms [3]-[5] and dynamic programming [6]-[8] have been proposed. This problem becomes more challenging when it comes to cognitive radio [9]. Some researchers have jointly considered the availability of channels with the video multicasting decisions through opportunistic multicasting approach [10], [11]. Nevertheless, most works focus on the static case. In practice, the SVC multicasting system will be highly dynamic in a long run. Therefore, there is a need of stochastic analysis. Plus, a commercial service provider will provide such a service only when significant economic value can be achieved [12]. Therefore, we discuss a subscriptionbased economic model where rational users' selfish nature is involved [13], [14]. The selfish behaviors may degrade the service provider's revenue [15]. We will use game theory to analyze how users react under the subscription model.

In light of these concerns, we study a dynamic SVC multicasting system with stochastic user arrival and heterogeneous user demand in this paper. Specifically, we propose a Multidimensional Markov Decision Process (M-MDP) framework to analyze the optimal load balancing and economic efficient policies for the system. The M-MDP framework is a stochastic extension [16] to the Chinese restaurant game [17], [18], in which the authors investigate how the negative network externality (resource allocation under heterogeneous demands in this work) influence the decisions of rational users. In this paper, we introduce a subscription system to help the service provider to regulate subscription requests from the heterogeneous users. Our main contributions are as follows:

1) We develop a Multi-dimensional Markov decision process based stochastic framework to analyze the resource allocation in a SVC multicasting system with heterogeneous user demands. Such a framework is more general than the existing snapshot-based approaches in the literature.

2) We study how rational users submit their demands under the subscription model through the proposed gametheoretic model. To the best of our knowledge, this paper is the first work bringing game theoretic analysis to the SVC multicast system.

3) We evaluate the economic value of the SVC multicasting system. We propose a dynamic iterative updating method to derive the optimal policy and pricing strategy.

\section{SYSTEM MODEL}

We consider a video multicasting server with multiple potential users who arrive and depart stochastically. The service 
provider determines the video server's resource allocation and the prices of the services, while the users request for the service when they arrive the system based on their preferences, computation capabilities, and the price of the service.

Video Server: Let us consider a SVC video server which is capable of serving at most $N$ users providing a set of $\mathcal{J}=$ $\{1,2, \ldots, J\}$ videos. Each SVC video contains $K$ layers. A user may decode the video up to layer $k$ only when all layer $1 \sim k$ are successfully received. The server transmits the layers of each video periodically on the same channel. All layers are transmitted with the same interval but at different period so they do not interfere with each other.

The reception of the layer is determined by two factors: the supported modulation and coding scheme (MCS) at the user side and the MCS applied on the layer at the video server side. On the user side, let $g_{i} \in \mathcal{G}$ be the maximum MCS supported by user $i$, where $\mathcal{G}$ is the universe set of the MCSs. In this paper, we assume that $g_{i}$ is a random variable with a probability density function $f\left(g_{i}\right)$. We assume the $g_{i}$ of each user $i$ is independent from each other and among time slots. On the video server side, let $g_{j, k}$ be the MCS applied on layer $k$ of video $j$. Then, only users with supported MCS $g \geq g_{j, k}$ can successfully receive this stream.

The applied MCS $g_{j, k}$ determines the required resource (transmission time) to transmit the layer. We denote $R_{j, k}\left(g_{j, k}\right)$ as the required resource to transmit layer $k$ of video $\bar{j}$ to users with MCS $g_{j, k}$. We then define $R_{j, k}\left(g^{c}\right) \equiv 0$. The $g^{c}$ represents the case that the transmission of this layer is disabled. Finally, let $R^{\text {total }}$ be the total available resource, which is the total transmission time in our system. We then have a $R^{\text {total }} \geq \sum_{j, k} R_{j, k}\left(\underline{g_{j, k}}\right)$.

Notice that the resource should be dynamically allocated according to the current loading. Let $\mathbf{s}=\left(n_{j, k}^{s} \mid j \in \mathcal{J}, k \in\right.$ $\{1 \ldots K\})$ be the current system state, where $n_{j, k}^{s}$ be the number of users requesting video $j$ 's layer $k$ stream. The state $\mathbf{s}$ represents the current loading of the video server. Then, we denote the dynamic resource allocation rule as $\mathbb{D}(\mathbf{s})=\left\{g_{j, k} \mid \forall j, k\right\}$. The allocation rule takes the system state $\mathbf{s}$ as input and outputs the corresponding MCS for each layer. In a utility-based system [3], [4], a common and reasonable choice of $\mathbb{D}$ is the overall utility maximization, that is,

$$
\mathbb{D}(\mathbf{s})=\arg \max _{\underline{g_{j, k}}} \mathbb{E}\left[\sum u_{i}\left(\left\{\underline{g_{j, k}}\right\}\right)\right]
$$

under the resource constraint $R^{\text {total }} \geq \sum_{j, k} R_{j, k}\left(g_{j, k}\right)$, where $u_{i}(\cdot)$ is the utility function of user $i$. In a wireless system with finite choices of the supported MCSs, this problem has been shown to be NP-hard [3]. Therefore, heuristic approaches are required and can be found in the literature [3]-[8].

User Valuation: Users with different preferences arrive stochastically. A user may prefer only a subset of all available videos. Plus, users with similar preferences usually have similar valuations on video content and quality. However, their devices may have different capabilities in receiving and decoding the videos. Therefore, users' abilities to have better video quality is limited by their equipment.
We model all the aforementioned properties with the following notations. Users are categorized into types, which is denoted by $t \in\{1,2, \ldots, T\}=\mathcal{T}$. A type $t=\left(\mathcal{J}^{t}, k^{t}\right)$ user prefers videos $j^{t} \in \mathcal{J}^{t}$ and is equipped with a device capable of decoding the SVC video up to layer $k^{t}$. We denote the valuation function on video $j \in \mathcal{J}$ with maximum decoded layer $k$ as $v_{j}(k)$. Then, a type $t$ user's valuation on video $j$ with maximum consecutively received layer $k$ is denoted as

$$
v^{t}(j, k)= \begin{cases}v_{j}\left(\min \left\{k, k^{t}\right\}\right), & j \in \mathcal{J}^{t} \\ 0, & \text { else. }\end{cases}
$$

Finally, as a stochastic model, we assume users with different types arrive independently. We describe the arrival process of type $t$ users with a Poisson process with the average arrival rate $\lambda^{t}$. Plus, the time period of a user staying in the system is an exponential process with the average departure rate $\mu$.

Payment System: We consider a subscription-based payment system as the revenue source of the service provider. A subscription contains two terms: the subscribed video $j$ and the desired maximum layer $k$. When a subscription is accepted by the video server, the required decryption keys of video $j$ 's layer $1 \sim k$ streams are delivered to the user. However, the receiving of these streams depends on $g_{j, k}$ and $g$, while the latter one is characterized by $f(g)$.

The price for a subscription, which is determined by the service provider, is modeled as a function of the system state s. We consider the per-slot charge scheme: whenever a user stays in the system with a subscription $(j, k)$, he is charged with a price of $P_{j, k}(\mathbf{s})$ where $\mathbf{s}$ is the current system state. Notice that a subscription will be halted when the user departs.

\section{GAME TheOreticAl Formulation}

In our model, we assume that users are rational and thus naturally selfish. Therefore, we need to consider users' selfish behaviors when evaluating and designing the pricing schemes and strategies by game theory [13], [14].

We consider a subscription game where the players are the potential users and the service provider. The service provider determines and announces at the beginning of the game the service price $\left\{P_{j, k}(\mathbf{s})\right\}$, while the potential users submit their requests after receiving the announcements from the service provider. The objective of the service provider is to maximize her expected revenue. In contrast, the objective of users are maximizing their own utility. As a result, the service provider needs to carefully determine the pricing strategy by taking into account the response of selfish users.

A user's objective is to maximize her own utility by choosing the best subscription. As described in Section II, users arrive stochastically. When a type $t$ user arrives, he determines whether to subscribe to the service with a specific video at certain layers. The expected utility of the user with subscription $(j, k)$ is conditioned on the system state $\mathbf{s}$ when he arrives. Let the system state at time slot $l$ be $\mathbf{s}^{l}$. A type $t$ user with a valid subscription $(j, k)$ has an immediate valuation on the service, $v^{t}(j, \bar{k})$, where $\bar{k} \leq k^{t}$ is the maximum successfully decoded layer at current time slot. In addition, 
there is a cost of using the service, which is the payment determined by price. Given the state and costs, the expected utility of a type $t$ user with a subscription $(j, k)$ is

$$
\mathbb{E}\left[u^{t}(j, k) \mid \mathbf{s}\right]=\sum_{l=l_{a}}^{l_{d}}\left(\mathbb{E}\left[v^{t}(j, \bar{k}) \mid \mathbf{s}=\mathbf{s}^{l}, \bar{k} \leq k\right]-P_{j, k}\left(\mathbf{s}^{l}\right)\right) .
$$

where $P_{j, k}\left(\mathbf{s}^{l}\right)$ is the per-slot charge pricing function, $\mathbf{s}$ is the state when the user arrives the system, and $l_{a}$ and $l_{d}$ are the user's arrival and departure time indices, respectively. A rational user will choose the subscription that maximizes the expected utility in (3) when arriving at the system.

\section{EQUILIBRIUM CONDITIONS}

Nash equilibrium is a solution concept for predicting the outcomes of a game [14]. It describes an action profile, where each player's action is the best response to other players' actions in the profile. The Nash equilibrium of the proposed subscription game can be analyzed as follows. We first model the selfish users' behaviors through a Multidimensional Markov decision process (M-MDP). The expected utility of users can then be calculated, and therefore the users' equilibrium conditions can be derived. We then derive the best responses for the service provider to maximize the revenue.

Multi-Dimensional Markov Decision Process: The video subscription game, when the pricing function $P_{j, k}(\mathbf{s})$ is given and fixed, can be formulated as a M-MDP. A traditional MDP describes a stochastic system where the state transition is (partially) regulated by a single decision maker. The decision maker aims to maximize her expected reward (utility). The optimal solution of MDP can be found by dynamic programming [19]. However, since there are multiple decision makers (users) with their own utilities in our game, the traditional MDP cannot be applied here. Instead, we propose a MMDP framework [16] with system state be $\mathbf{s} \in \mathcal{S}$. Since the server serves up to $N$ users, we have the boundary constraints $\sum_{j, k} n_{j, k}^{s} \leq N$ on the universe state set $\mathcal{S}$.

The action of a user is the subscription request $a=(j, k)$. Different types of users may have different action space due to the limitation in computation capability. The action space of a type $t$ user is $\mathcal{A}^{t}=\mathcal{J}^{t} \times \mathcal{K}^{t} \bigcup\{(0,0)\}$, where $(0,0)$ represents that he does not subscribe to any video and leaves the system immediately. Note that users will not subscribe the unpreferred videos since their valuations on those videos are zero. Therefore, we denote $V(j, k) \equiv v_{j}(k)$ to describe any user's valuation on the video if he indeeds submit a request $(j, k)$. Therefore, after taking the action $a=(j, k)$, the user can obtain an immediate reward as follows

$$
R(\mathbf{s}, j, k)=\mathbb{E}[U(j, k) \mid \mathbf{s}]=V_{j, k}(\mathbf{s})-P_{j, k}(\mathbf{s}) .
$$

where $U(j, k)$ is the common utility function of any user in the system if he indeeds request for $(j, k)$. In general, the immediate reward is the expected valuation of the successfully decoded video $V_{j, k}(\mathbf{s})$ minus the subscription payment, which happens to be the expected utility of a type $t$ user with subscription $\left(j \in \mathcal{J}^{t}, k\right)$ in state $\mathbf{s}$. For the case that $(j, k)=(0,0)$, we let $R(\mathbf{s}, 0,0)=0, \forall \mathbf{s}$.

A policy is an action profile which describes a decision of the decision maker at a certain state. In our model, a policy is denoted as a function $\pi(\mathbf{s}, t): \mathcal{S} \times \mathcal{T} \mapsto \mathcal{A}^{t}$, which describes the subscription decision of type $t$ user arriving in state $\mathbf{s}$.

Expected Reward under Transition Probability: A rational user will make the decision to maximize the expected reward defined as follows

$$
W(\mathbf{s}, j, k)=\mathbb{E}\left[\sum_{l=l^{e}}^{\infty}(1-\mu)^{t-1} R\left(\mathbf{s}^{l}, j, k\right) \mid \mathbf{s}\right]
$$

where $\mu$ is the departure rate, and thus $1-\mu$ is the probability that the user will stay at next time slot. Note that $1-\mu$ can also be considered as the discount factor of the future utility. In the steady state, the Bellman equation of the expected reward is

$W(\mathbf{s}, j, k)=R(\mathbf{s}, j, k)+(1-\mu) \sum \operatorname{Pr}\left(\mathbf{s}^{\prime} \mid \mathbf{s}, \pi, j, k\right) W\left(\mathbf{s}^{\prime}, j, k\right)$

where $\operatorname{Pr}\left(\mathbf{s}^{\prime} \mid \mathbf{s}, \pi, j, k\right)$ denotes the transition probability from $\mathbf{s}$ to $\mathbf{s}^{\prime}$ when the user takes the action $(j, k)$ under the policy $\pi$. Notice that this transition probability is conditioned on the fact that he is not leaving the system at next time slot. Otherwise he will not receive the rewards from next time slot. Let $\mathbf{e}_{j, k}$ be a standard basis vector. Then, the transition probability of a user with a subscription $(j, k)$ is given as follows

$$
\begin{aligned}
& \operatorname{Pr}\left(\mathbf{s}^{\prime} \mid \mathbf{s}, \pi, j, k\right) \\
& = \begin{cases}\lambda^{t}, & \mathbf{s}^{\prime}=\mathbf{s}+\mathbf{e}_{\pi(\mathbf{s}, t)} ; \\
\left(n_{j^{\prime}, k^{\prime}}^{s}\right) \mu, & \mathbf{s}^{\prime}=\mathbf{s}-\mathbf{e}_{j^{\prime}, k^{\prime}} ; \\
\left(n_{j, k}^{s}-1\right) \mu, & \mathbf{s}^{\prime}=\mathbf{s}-\mathbf{e}_{j, k}, n_{j, k}^{s^{\prime}} \neq 0 ; \\
1-\mu(N(\mathbf{s})-1)-\lambda(\mathbf{s}, \pi), & \mathbf{s}=\mathbf{s}^{\prime}, n_{j, k}^{s^{\prime}} \neq 0 ; \\
1-\mu N(\mathbf{s})-\lambda(\mathbf{s}, \pi), & \mathbf{s}=\mathbf{s}^{\prime}, n_{j, k}^{s^{\prime}}=0 ; \\
0, & \text { else. }\end{cases}
\end{aligned}
$$

where $\lambda(\mathbf{s}, \pi)=\sum_{t \in \mathcal{T}} \mathbf{1}(\pi(\mathbf{s}, t) \neq(0,0)) \lambda^{t}$ and $N(\mathbf{s})=$ $\sum_{j, k} n_{j, k}^{s}$. Since users are assumed to be rational, a type $t$ user should choose the subscription that maximizes her expected utility when he arrives at the system. The optimal policy under the expected reward $W(\mathbf{s}, j, k)$ is given by

$$
\pi(\mathbf{s}, t) \in \arg \max _{(j, k) \in \mathcal{A}^{t}} W\left(\mathbf{s}+\mathbf{e}_{j, k}, j, k\right),
$$

Notice that when the server is full, new users will not be allowed to enter, i.e., $\forall N(\mathbf{s})=N, \pi(\mathbf{s}, t)=(0,0), \forall t \in \mathcal{T}$.

The Nash equilibrium is achieved when (6) and (8) are satisfied, which are denoted as the equilibrium conditions. When the equilibrium conditions are met, each type of users has chosen the subscription that maximizes the expected utility. Therefore, a Nash equilibrium is achieved.

Average Revenue Maximization: The objective of the service provider is to maximize her expected revenue under the response of users. Let $\pi$ and $W$ be the policy and expected rewards derived in (6) and (8) under the pricing function $P_{j, k}(\mathbf{s})$. Then, let $Q(\mathbf{s})$ be the expected revenue in state $\mathbf{s}$. The best response of the service provider is the solution to: $\max _{P_{j, k}(\mathbf{s})} \lim _{N \rightarrow \infty} \frac{1}{N} \mathbb{E}\left[\sum_{l=1}^{N} Q\left(\mathbf{s}^{l}\right)\right]$, respect to 
$W^{*}\left(\mathbf{s}+\mathbf{e}_{\pi^{*}(\mathbf{s}, t)}, \pi^{*}(\mathbf{s}, t)\right) \geq 0, \forall \mathbf{s}, t$, where $\pi^{*}$ and $W^{*}$ satisfy (6) and (8). Note that the state transition probability in this problem is different from the one observed by users in (7), and can be written as follows

$$
\operatorname{Pr}\left(\mathbf{s}^{\prime} \mid \mathbf{s}, \pi\right)= \begin{cases}\lambda^{t}, & \mathbf{s}^{\prime}=\mathbf{s}+\mathbf{e}_{\pi(\mathbf{s}, t)} \\ \left(n_{j, k}^{s}\right) \mu, & \mathbf{s}^{\prime}=\mathbf{s}-\mathbf{e}_{j, k} \\ 1-N(\mathbf{s}) \mu-\lambda(\mathbf{s}, \pi), & \mathbf{s}=\mathbf{s}^{\prime} \\ 0, & \text { else. }\end{cases}
$$

With primal-dual transformation [19], the expected average revenue can be given by $g(\pi)=\sum_{\mathbf{s} \in \mathcal{S}} \operatorname{Pr}(\mathbf{s} \mid \pi) Q(\mathbf{s})$, where $\operatorname{Pr}(\mathbf{s} \mid \pi)$ is the stationary state distribution under policy $\pi$.

\section{Optimal Pricing Strategy and Policy}

Optimal Pricing Strategy: First, we would like to discuss the optimal pricing strategy that maximizes the expected revenue under a given policy $\pi$ while satisfying the equilibrium conditions in (6) and (8). Since the policy is given and fixed, the optimization problem is simplified. This helps us to understand how the service provider should determine the price in order to maximize her expected revenue given a known pattern on the subscription requests from users.

Let $\operatorname{Pr}(\mathbf{s} \mid \pi)$ be the stationary probability that the system is in state $\mathbf{s}$ under policy $\pi$, which is given. Then, the original revenue maximization problem can be written as

$$
\max _{\left\{P_{j, k}(\mathbf{s})\right\}} \sum_{\mathbf{s} \in \mathcal{S}} \operatorname{Pr}(\mathbf{s} \mid \pi) \sum_{j \in \mathcal{J}, k \in \mathcal{K}} n_{j, k}^{s} P_{j, k}(\mathbf{s})
$$

under the constraints in (8). Then, let $\mathcal{R}_{j, k}, \mathcal{W}_{j, k}, \mathcal{V}_{j, k}$, and $\mathcal{P}_{j, k}$ be the $1-$ by- $|\mathcal{S}|$ matrix of $R(\mathbf{s}, j, k), W(\mathbf{s}, j, k) V_{j, k}(\mathbf{s})$, and $P_{j, k}(\mathbf{s})$ over $\mathcal{S}$. Then, let $\mathcal{L}(\pi)$ be the state transition probability matrix, which is a $|\mathcal{S}|-$ by $-|\mathcal{S}|$ matrix with terms $\operatorname{Pr}\left(\mathbf{s}^{\prime} \mid \mathbf{s}, \pi\right)$ over $\mathcal{S}$. From (4) and (6), we have

$$
(I-(1-\mu) \mathcal{L}(\pi)) \mathcal{W}_{j, k}=\mathcal{R}_{j, k}=\mathcal{V}_{j, k}-\mathcal{P}_{j, k}, \forall j, k
$$

Therefore, the constraints in (8) can be re-written as

$$
\begin{aligned}
& (I-(1-\mu) \mathcal{L}(\pi))^{-1}\left(\mathcal{V}_{j, k}-\mathcal{P}_{j, k}\right)=\mathcal{W}_{j, k},, \forall j, k \\
& W\left(\mathbf{s}+\mathbf{e}_{\pi(\mathbf{s}, t)}, \pi(\mathbf{s}, t)\right) \geq 0, \forall \pi(\mathbf{s}, t) \neq(0,0) \\
& \left.W\left(\mathbf{s}+\mathbf{e}_{\pi(\mathbf{s}, t)}\right), \pi(\mathbf{s}, t)\right)-W\left(\mathbf{s}+\mathbf{e}_{j, k}, j, k\right) \geq 0 \\
& \forall \pi(\mathbf{s}, t) \neq(0,0),(j, k) \in \mathcal{A}^{t} \\
& W\left(\mathbf{s}+\mathbf{e}_{\pi(\mathbf{s}, t)}, \pi(\mathbf{s}, t)\right) \leq 0, \forall \pi(\mathbf{s}, t)=(0,0),(j, k) \in \mathcal{A}^{t}
\end{aligned}
$$

which is a set of linear constraints over $\mathcal{P}=\left\{P_{j, k}(\mathbf{s}) \mid \mathbf{s} \in\right.$ $\mathcal{S}, j \in \mathcal{J}, k \in \mathcal{K}\}$. Therefore, the original problem is equivalent to the following linear programming problem:

$$
\max _{\mathcal{P}} \sum_{\mathbf{s} \in \mathcal{S}} \operatorname{Pr}(\mathbf{s} \mid \pi) \sum_{j \in \mathcal{J}, k \in \mathcal{K}} n_{j, k}^{s} P_{j, k}(\mathbf{s})
$$

with the constraints in (11) - (14), which can be solved by standard linear programming methods.

Revenue-Maximized Policy: Finding revenue-maximized policy is challenging since the policy, revenue function and the pricing strategy couple together. In such a case, the traditional MDP solvers cannot be directly applied for the revenuemaximization MDP problem. Let $\mathcal{L}(\pi)$ be the state transition
TABLE I

VIDEO SPECIFICATIONS

\begin{tabular}{|c|c||c|c||c|c|}
\multicolumn{4}{c}{ MOBCAL } & \multicolumn{2}{c|}{ STOCKHOLM } \\
\hline Layer & Res. & Bit-Rate & $v(k)$ & Bit-Rate & $v(k)$ \\
\hline 1 & $360 \mathrm{i}$ & $315.33 \mathrm{kbps}$ & 0.5 & $319.75 \mathrm{kbps}$ & 0.8 \\
1,2 & $720 \mathrm{i}$ & $1660.83 \mathrm{kbps}$ & 0.75 & $1480.32 \mathrm{kbps}$ & 0.9 \\
$1,2,3$ & $1080 \mathrm{i}$ & $10719.41 \mathrm{kbps}$ & 1 & $6806.29 \mathrm{kbps}$ & 1 \\
\hline
\end{tabular}

TABLE II

USER SPECIFICATIONS

\begin{tabular}{|c|c|c|}
\hline Type & Preferred Video(s) & Maximum Layer \\
\hline 1 & MOBCAL & 2 \\
2 & STOCKHOLM & 2 \\
3 & STOCKHOLM & 3 \\
4 & MOBCAL,STOCKHOLM & 3 \\
\hline
\end{tabular}

matrix and $Q^{*}(\mathbf{s}, \pi)=\sum_{j \in \mathcal{J}, k \in \mathcal{K}} n_{j, k}^{s} P_{j, k}(\mathbf{s})$ be the revenue vector over states, where $P_{j, k}(\mathbf{s})$ is the solution to (15). Then, the revenue optimization problem becomes

$$
\max _{\pi} \lim _{N \rightarrow \infty} \frac{1}{N} \sum_{l=1}^{N} \mathcal{L}(\pi)^{l-1} Q^{*}\left(\mathbf{s}^{\mathbf{0}}, \pi\right),
$$

which is a average-reward Markov decision process. Nevertheless, the immediate reward $\mathcal{V}^{*}$ depends on the policy $\pi$, due to which, traditional algorithms for MDP cannot be applied here. A dynamic iterative updating approach can be applied here to find the solution by updating the $\mathcal{V}^{*}$ with solution to (15) and $\pi$ using the policy iteration algorithm iteratively.

\section{Simulation Results}

We consider a SVC multicasting service over a WiMAX network with the same wireless system parameters described in [3], where 7 level of MCSs in WiMAX standard are chosen. The channel quality is quantized to corresponding seven levels $\mathcal{G}=\{1,2,3,4,5,6,7\}$. In all simulations, we assume each user's channel quality $g$ is uniformly distributed in these seven levels. A data stream with a bit-rate of $R$ requires $R / r_{m}$ time per second to transmit if MCS $m$ is chosen, where $r_{m}$ is the data rate of MCS $m$. The allocation rule $\mathcal{D}$ is maximizing the overall valuation given the demands from users. The allocation is derived through exhaustive search.

The server can serve up to 12 users, while the service time per second is a ratio between $0 \%$ and $100 \%$. The server provides two videos, MOBCAL and STOCKHOLM [20]. We use JSVM [21] to encode each video into a 3-layer spatialscaled H.264 SVC stream, where the specs are listed in Table I. There are four types of users specified in Table II. The user's valuations on videos are shown in Table I. Finally, the user arrival and departure parameters are set to be 0.04 and 0.01 .

We evaluate the system efficiency through two performance metrics: average social welfare, which is the total users' valuations on the service, and average revenue. For the social welfare performance, we compare two policies: revenuemaximized policy from Section $\mathrm{V}$, and free subscription policy. The latter one is the solution to (6) and (8) with $P_{j, k}(\mathbf{s})=0, \forall \mathbf{s}$. By comparing these two policies, we can evaluate the efficiency loss when no pricing scheme is applied. For the expected revenue, we compare two pricing schemes: optimal per-slot charge and fixed entrance fee. The former one 

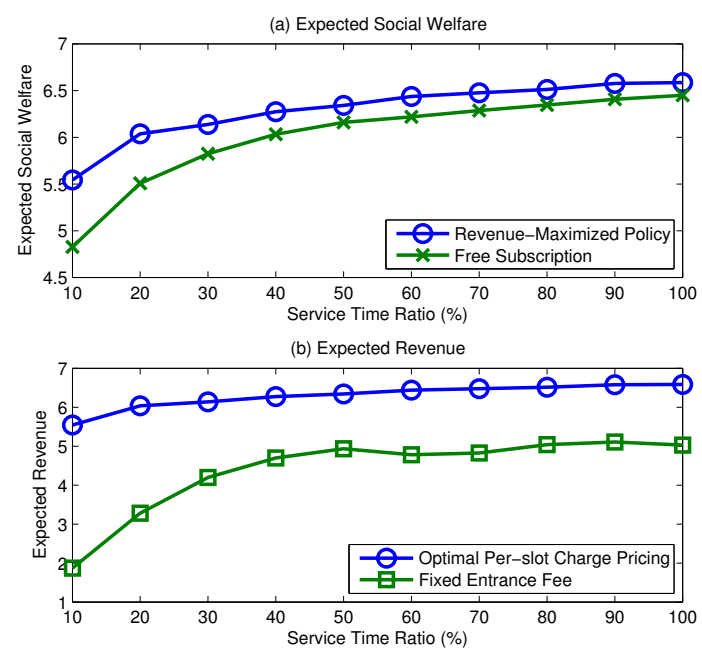

Fig. 1. System Performance under Different Available Service Time Ratio

is the proposed pricing scheme, while the latter one is a fixed entrance fee for every subscription, which is widely adopted in current video subscription services. The entrance fee in the scheme is chosen as $P^{e}=\min _{\mathbf{s}, t, \pi(\mathbf{s}, t) \neq(0,0)} W^{i n d v}(\mathbf{s}+$ $\left.\mathbf{e}_{\pi(\mathbf{s}, t)}, \pi(\mathbf{s}, t)\right)$, where $W^{i n d v}$ is the expected reward under the free subscription policy.

We investigate how the amount of service time affects the efficiency of the SVC multicasting system. We control the service time ratio from 0 to $100 \%$ with other settings unchanged. The results are shown in Fig. 1. We first observe that the revenue-maximized policy always achieves higher social welfare than the free subscription policy (Fig. 1(a)). For the average revenue, we observe that the optimal pricing scheme has higher revenue than the fixed entrance fee (Fig. 1(b)). Notice that the revenue under fixed entrance fee does not always increase when the service time ratio increases. This is because that the optimal resource allocation problem in (1) is nonlinear. When the service time increases, some streams may eventually get less resource in order to improve the transmission quality of other streams along with the increased resource under the optimal allocation. In such a case, the fixed entrance fee scheme has a lower revenue since the price under this scheme is constrained by the video stream with lowest expected reward. Nevertheless, our proposed pricing scheme is resistant to this effect since the expected revenue under our pricing scheme is equal to the overall social welfare, which is nondecreasing with the increases of service time.

\section{CONCLUSIONS}

We proposed a novel framework to study a general stochastic SVC multicasting system. This framework supports stochastic user arrival and heterogeneous user preferences and is applicable to existing resource allocation algorithms in the literature. A subscription-based payment system was studied in this framework for exploring the economic value of the system with rational users. The responses of selfish users under the per-slot charge scheme was discussed and the equilibrium conditions were derived as the constraints of the corresponding revenue optimization problem for the service provider. The optimal pricing strategies and policies which maximize the expected revenue of the system can be derived by dynamic iterative updating techniques. We showed that the revenuemaximized policy is a socially-optimal policy, which means that the proposed optimal policy also maximizes the social welfare.

\section{ACKNOWLEDGMENT}

Chih-Yu Wang and Hung-Yu Wei are in part supported by National Science Counsel Grant 101-2219-E-002-003- and the Ministry of Economic Affairs Grant 101-EC-17-A-03-S1-214.

\section{REFERENCES}

[1] H. Schwarz, D. Marpe, and T. Wiegand, "Overview of the Scalable Video Coding Extension of the H.264/AVC Standard," IEEE Trans. on Circuits and Systems for Video Technology, vol. 17, no. 9, pp. 1103 -1120 , Sep. 2007.

[2] S.-F. Chang and A. Vetro, "Video Adaptation: Concepts, Technologies, and Open Issues," Proceedings of the IEEE, vol. 93, no. 1, pp. $148-158$, Jan. 2005

[3] W.-H. Kuo, W. Liao, and T. Liu, "Adaptive Resource Allocation for Layer-Encoded IPTV Multicasting in IEEE 802.16 WiMAX Wireless Networks," IEEE Trans. on Multimedia, vol. 13, no. 1, pp. 116-124, Feb. 2011.

[4] S. Deb, S. Jaiswal, and K. Nagaraj, "Real-Time Video Multicast in WiMAX Networks," in IEEE INFOCOM, Phoenix, AZ, USA, Apr. 2008.

[5] Y. Yu, P. Hsiu, and A. Pang, "Energy-Efficient Video Multicast in 4G Wireless Systems," IEEE Trans. on Mobile Computing, to appear.

[6] P. Li, H. Zhang, B. Zhao, and S. Rangarajan, "Scalable Video Multicast With Adaptive Modulation and Coding in Broadband Wireless Data Systems," IEEE-ACM Trans. on Networking, vol. 20, no. 1, pp. 57-68, Feb. 2012.

[7] S. Chuah, Z. Chen, and Y. Tan, "Energy-Efficient Resource Allocation and Scheduling for Multicast of Scalable Video over Wireless Networks," IEEE Trans. on Multimedia, no. 4, pp. 1324-1336, Aug. 2012.

[8] W. Ji, Z. Li, and Y. Chen, "Joint Source-Channel Coding and Optimization for Layered Video Broadcasting to Heterogeneous Devices," IEEE Trans. on Multimedia, vol. 14, no. 2, pp. 443-455, Apr. 2012.

[9] Y. Chen, Y. Wu, B. Wang, and K. J. R. Liu, "Spectrum Auction Games for Multimedia Streaming over Cognitive Radio Networks," IEEE Trans. on Communications, vol. 58, no. 8, pp. 2381-2390, Aug. 2010.

[10] P. Polacek, T.-Y. Yang, and C.-W. Huang, "Joint Opportunistic Spectrum Access and Scheduling for Layered Multicasting over Cognitive Radio Networks," in IEEE MMSP, Hangzhou, China, Oct. 2011.

[11] C.-W. Huang, S.-M. Huang, P.-H. Wu, S.-J. Lin, and J.-N. Hwang, "OLM: Opportunistic Layered Multicasting for Scalable IPTV over Mobile WiMAX," IEEE Trans. on Mobile Computing, vol. 11, no. 3, pp. $453-463$, Jan. 2012.

[12] P.-H. Wu and Y. Hu, "Optimal Layered Video IPTV Multicast Streaming Over Mobile WiMAX Systems," IEEE Trans. on Multimedia, vol. 13, no. 6, pp. 1395-1403, Dec. 2011.

[13] Y. Chen and K. J. R. Liu, "Understanding Microeconomic Behaviors in Social Networking: An engineering view," IEEE Signal Processing Magazine, vol. 29, no. 2, pp. 53 -64, Mar. 2012.

[14] B. Wang, Y. Wu, and K. J. R. Liu, "Game Theory for Cognitive Radio Networks: An Overview," Computer Networks, vol. 54, no. 14, pp. 2537-2561, Oct. 2010.

[15] W. Lin and K. J. R. Liu, "Game-Theoretic Pricing for Video Streaming in Mobile Networks," IEEE Trans. on Image Processing, vol. 21, no. 5, pp. $2667-2680$, May 2012.

[16] C. X. Jiang, Y. Chen, Y. Yang, C. Wang, and K. J. R. Liu, "Dynamic Chinese Restaurant Game in Cognitive Radio Networks," in IEEE INFOCOM, Turin, Italy, April 2013.

[17] C.-Y. Wang, Y. Chen, and K. J. R. Liu, "Chinese Restaurant Game," IEEE Signal Processing Letters, vol. 19, no. 12, pp. 898 -901, Dec. 2012.

[18] - "Sequential Chinese Restaurant Game," IEEE Trans. on Signal Processing, vol. 61, no. 3, pp. 571 -584, Feb. 2013.

[19] M. L. Puterman, Markov Decision Processes: Discrete Stochastic Dynamic Programming, 1st ed. John Wiley \& Sons, Inc., 1994.

[20] L. Haglund, "SVT Video Test Sequence," SVT Sveriges Television $A B$, 2001. [Online]. Available: ftp://ftp.ldv.e-technik.tu-muenchen.de/ pub/test_sequences/

[21] H. A. Q. Maarif, T. S. Gunawan, and O. O. Khalifa, "JSVM Reference Software." 